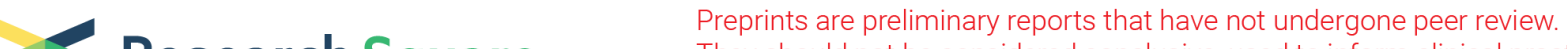 Research Square They should not be considered conclusive, used to inform clinical practice, or referenced by the media as validated information.
}

\section{Sustainable power generation for at least one month from ambient humidity using unique nanofluidic diode}

\section{Yong Zhang}

Southwest Jiaotong University

Tingting Yang ( $\nabla$ yangtingting@swjtu.edu.cn )

Southwest Jiaotong University https://orcid.org/0000-0001-6773-8438

\section{Kedong Shang}

Southwest Jiaotong University

\section{Fengmei Guo}

Zhengzhou University

\section{Yuanyuan Shang}

Zhengzhou University

\section{Shulong Chang}

Zhengzhou University

\section{Licong Cui}

Southwest Jiaotong University

\section{Xulei Lu}

Southwest Jiaotong University

Jian Zhou

Southwest Jiaotong University

\section{Chunqiao Fu}

Southwest Jiaotong University

\section{Qichang He}

University of Paris-Est

\section{Article}

Keywords: energy-harvesting of humidity, clean energy source, sustainable power generation

Posted Date: November 24th, 2021

DOI: https://doi.org/10.21203/rs.3.rs-1084515/v1 
License: (c) (i) This work is licensed under a Creative Commons Attribution 4.0 International License. Read Full License

Version of Record: A version of this preprint was published at Nature Communications on June 16th, 2022. See the published version at https://doi.org/10.1038/s41467-022-31067-z. 


\section{Abstract}

The continuous energy-harvesting of humidity naturally present in air is attractive for the development of clean energy source for self-sustained systems. Controlling the transport of ionized mobile charge in intelligent nanoporous membrane systems is a promising strategy to convert ambient moisture energy to electricity. However, existing designs still suffer from low output power density. Moreover, these devices can only produce short-term (mostly a few seconds or a few hours, rarely for a few days) voltage and current output in the ambient environment. Effort devoted to pursuing membrane materials with new sustained energy conversion mechanism is urgently needed. Here, we design an ionic diode-type hybrid membrane with carbon nanotube/anodized aluminum asymmetric structure capable of continuously harvesting moisture energy in the ambient environment. Under electric fields, a hybrid membrane exhibits ionic current rectification properties, enabling high energy conversion efficiency in the long run due to steady-state one-way ion transport. Using a synergy between the ion rectification of nanofluidic diode and the built-in electric field induced ionization and charge transfer, one single unit produces a sustained maximum open-circuit-voltage, short-circuit-current, short-circuit-current density, output power density of $1.1 \mathrm{~V}, 7.7 \mu \mathrm{A}, 11.3 \mu \mathrm{Acm}^{-2}$ and $1.3 \mu \mathrm{Wcm}^{-2} / 277 \mu \mathrm{Wcm}^{-3}$, respectively $\left(93 \% \mathrm{RH}, 25^{\circ} \mathrm{C}\right)$. Besides, the strong hydrogen bonds and van der Waals forces formed at the interface of asymmetric structure make the hybrid membrane robust in humid environment. In particular, the strategy of adjusting the moisture-based energy-harvesting performance based on the rectification effect of the nanofluidic diode is reported for the first time. Compared with the relevant existing devices, those elaborated by the present work show the longest service life and can generate continuous voltage and current for at least one month, demonstrating the feasibility of long-term power generation in any location with moisture conditions.

\section{Introduction}

In recent years, water-based power generators have become a promising power generation technology due to the abundance, cleanliness and sustainability of water. Numerous water-powered generators using pure liquid water and aqueous solution as energy source have emerged ${ }^{1-7}$, relying on streaming potential $^{8-13}$, dragging potential ${ }^{14-16}$, waving potential ${ }^{17-20}$ and triboelectric potential ${ }^{1,20-22}$ etc. However, these devices require continuous or periodic water supplementation, which limits their installation location for practical use. As one form of water, moisture is abundantly present in air. The ubiquity of atmospheric moisture makes the development of moisture-based energy-harvesting technologies promising for solving the energy problem of low-power electronics and loT (Internet of things) devices ${ }^{23-26}$. In one early strategy, water vapor adsorption on porous carbon film with a nonhomogeneous vertical distribution of carboxy groups can generate a concentration gradient of the released $\mathrm{H}^{+}$ions ${ }^{27-29}$. But after a short duration of power output $(\sim 600 \mathrm{~s})^{29}$, the device voltage and current collapse since ionized mobile charge diffusion gradually reaches equilibrium, terminating the generation of electricity. Desirably, the power output should not be a transient phenomenon. However, a continuous power output relying on ambient atmospheric moisture remains challenging. 
Some innovative chemical and structural designs have been proposed to satisfy requirements for continuous electric output. A typical strategy is based on nanofluidic devices, because confined nanospace and capillaries are sensitive to external stimuli ${ }^{30-32}$ and can interact with water through many unique phenomena such as electric double layer coupling. For example, a power generator using protein nanowires film adopts the process of continuous exchange of water molecules at the solid interfaces to build a self-maintained moisture gradient ${ }^{33}$. Under the moisture gradient, nanowires with a high density of nanometre-scale pores and surface functional groups facilitate ionization and charge transfer for continuous electric output. Indeed, the open-circuit voltage $\left(V_{O C}\right)$ and short-circuit current $\left(I_{S C}\right)$ of around $0.5 \mathrm{~V}$ and $250 \mathrm{nA}$ are generated. Significantly, the devices maintain a continuous $\mathrm{V}_{\mathrm{OC}}$ of $0.4-0.6 \mathrm{~V}$ for more than 2 months and a continuous current for at least 20 hours before self-recharging. Transpirationdriven electrokinetic power generator adopting a hydrological cycle with the surrounding air is another example ${ }^{34}$. The incorporation of $\mathrm{CaCl}_{2}$ to collect water vapor from the surrounding environment is crucial to acquiring a stable water supply to form the wet side of a carbon film. The water evaporation facilitates capillary flow from the wet to dry side, which induces a pseudo-streaming current. Meanwhile, a vertical setup causes the gradient distribution of $\mathrm{CaCl}_{2}$ content by gravity. Thus, two asymmetries, i.e., protons and $\mathrm{Ca}^{2+}$ ions, of the conductive nanoporous carbon surfaces are established, driving a continuous electrical output for at least 10 days. The devices exhibit maximum $\mathrm{V}_{\mathrm{OC}}(0.74 \mathrm{~V}), \mathrm{I}_{\mathrm{SC}}(22.5 \mu \mathrm{A})$ and electric power $(2.02 \mu \mathrm{W})$ when the film size is $3 \mathrm{~cm} \times 9 \mathrm{~cm} \times 0.12 \mathrm{~mm}$.

In addition to common strategies such as chemical modification and microstructure control, some new materials have also been introduced into the field of moisture-based power generation. Polyelectrolyte, which releases free ions (such as protons) under moisture, has been explored as one type of efficient moist-electric generating material. When one side of the polyelectrolyte membrane is under constant moisture feeding, protons gradually migrate to the other side under the proton concentration gradient, offering a maximum $\mathrm{V}_{\mathrm{OC}}(0.8 \mathrm{~V})$, I $\mathrm{SC}$ density $\left(100 \mu \mathrm{A} / \mathrm{cm}^{2}\right)^{35}$. However, for long-term measurement, the electric output drops back to zero after 2 days due to the homogeneity of water molecules within the membrane. Bilayer of polyelectrolyte film with heterogeneous distribution of charged mobile ions in moist air can extend the working time of one single device to at least 250 hours with $\mathrm{V}_{\mathrm{OC}}$ of $0.95 \mathrm{~V}$ under $25 \%$ $\mathrm{RH}^{36}$. Large-scale integration of abundant generator units is even able to offer a $\mathrm{V}_{\mathrm{OC}}$ of more than 1,000 V. However, the generated current output during 150 hours shows an obvious decrement (40nA at the beginning, $6 \mathrm{nA}$ after $6 \mathrm{~h}$, and $2 \mathrm{nA}$ after $150 \mathrm{~h}$ ).

In regard to daily electronic appliances, the life span of several months to several years is the threshold, which requires longer voltage and current output to meet applications in various fields. However, the performance summary of the existing humidity-enabled electric generator (HEEG) is shown in table S3, and simultaneous continuous voltage/current output for more than one month has not been realized. Therefore, obtaining membrane materials with new sustained energy conversion mechanism giving rise to high output power density and long-term stability is an urgent need. Solar photovoltaic power generation is a power generation method that uses the principle of photovoltaic effect to directly convert 
solar radiant energy into electrical energy. A typical solar cell is based on the PN junction semiconductor diode. After the photogenerated electron-hole pairs are generated in the barrier region of the PN junction, they are immediately separated by the built-in electric field. The photogenerated electrons are sent to the $\mathrm{N}$ zone, and the photogenerated holes are sent to the $\mathrm{P}$ zone, so that light energy is transformed into electrical energy. Up to now, HEEG devices often rely on nanofluid structure, in which positive and negative ions generated during water cycle transportation experience differential charge transfer, leading to hydrovoltaic effect. However, the selective separation of ions by only relying on Debye shielding effect is limited. Inspired by the form of the semiconductor PN junction in photovoltaic device, we herein propose a HEEG device by adopting ionic diode-type PN junction in moist air, as shown in Fig. 1a. Besides, the strategy adopting nanofluidic devices with rectified ionic transportation has been verified as very feasible both by theory and experiment in hypersaline environment to build an osmotic power generator system ${ }^{37,38}$. But how to use the ion rectification phenomenon in atmospheric moisture environment still remains a challenge. Herein, an ionic diode-type device consisting of a nanoporous carbon nanotubes (CNT) membrane and a nanochannel anodic aluminum oxide (AAO) membrane for moisture-based energy harvesting is developed as shown in Fig. 1b. The designed device holds the following five salient features:

i. The built-in potential formed in the asymmetric nanofluidic junction (humidity-diode 1, name style refers to photo-diode) facilitates ionization of adsorbed water molecules and one-way transfer of ion charge, thus causing power generation with less energy loss during the conversion process.

ii. AAO has sufficient surface charge density and adjusted pore size of dozens to hundreds of nanometers. In asymmetric moisture environment, AAO attracts ions with the opposite charge into the pores and squeezes out the same charge due to Debye screening effect. Such nanopore rectification process is equivalent to diode 2 . Moreover, ordered AAO fluid channel lowers the transport resistance of the ion charge, thus further improving the output power.

iii. Water molecules continuously adsorb, ionize, and selectively transport due to the synergy between Diode 1 and Diode 2, guaranteeing a continuous flow of ion charge which boosts sustainable power output after an effective conversion between ion current and electron current.

iv. The strong hydrogen bonds and van der Waals forces formed at the CNT/AAO interface endow the hybrid membrane with excellent robustness in humid environment, and such robust structure enables steady-state ion transportation and guarantees the long-term stability 39,40 .

v. CNT and AAO are two of the most used materials in various fields and can be easily fabricated in large-scale, which promotes their practical application.

Such a nanofluidic diode-based single unit can deliver a $\mathrm{V}_{\mathrm{OC}}$ of $1.1 \mathrm{~V}$ and an $\mathrm{I}_{\mathrm{SC}}$ of $7.7 \mu \mathrm{A}$ under $93 \% \mathrm{RH}$, $25^{\circ} \mathrm{C}$. The maximum short-circuit-current density is $11.3 \mu \mathrm{Acm}^{-2}$ when reducing the membrane size to $1 \mathrm{~mm}^{2}$, and the corresponding output power density is $1.3 \mu \mathrm{Wcm}^{-2} / 277 \mu \mathrm{Wcm}^{-3}$. In longer-term monitoring, our fabricated membrane shows a stable $\mathrm{V}_{\mathrm{OC}}$ of $0.8-1.1 \mathrm{~V}$ for at least a month, and $\mathrm{I}_{\mathrm{SC}}$ undergoes gradual attenuation from $1500 \mathrm{nA}$ to $100 \mathrm{nA}$ after 1 month. The degradation in current is possibly associated with 
the loss of oxide groups of CNT in the ambient relative humidity in the laboratory environment. Besides, the power supply of low-power LED devices and the self-powered respiratory monitoring device have also been explored using this power generation technology.

\section{Results And Discussions}

\section{Design, fabrication and characterization of ionic diode-type hybrid membrane}

The experimental configuration for electricity-generating devices from ambient humidity based on ionic diode-type hybrid membrane is illustrated in Fig. 1c. The photo of the device with a working area of 0.95 $\mathrm{cm}^{2}$ is shown in Fig. S1a. For detailed experimental procedures, please refer to the experimental section in the supplementary materials. Oxygen plasma treated carbon nanotube (CNT) thin film and liquid metal (Gallium Indium alloy) (Fig. S2) were stamped and blade-coated on the front and rear side of anodized aluminum (AAO) membrane as top and bottom electrodes, respectively. As shown in the scanning electron microscope (SEM) images of the CNT film in Fig. 1d and Fig. S1b, the CNT film prepared by chemical vapor deposition is mainly composed of interwoven carbon nanotube bundles, and the diameter of the tube bundles is mostly distributed between $10 \mathrm{~nm}$ and $30 \mathrm{~nm}$. There are many pores in the CNT film, which allow good moisture permeability, and the pore structure of the AAO film underneath is clearly visible in the SEM photo (Fig. S1b). An atomic force microscope (AFM) was used to measure the thickness of the carbon nanotube film transferred to the silicon wafer (Fig. S3). The thickness of CNT film is very thin, about tens to hundreds of nanometers. At the same time, the CNT film has excellent conductivity, and its square resistance is $\sim 400 \Omega$ when the thickness is $140 \mathrm{~nm}$ (Fig. S4). The CNT film is relatively soft and can be closely and conformally attached to a 47-micron thick AAO substrate to form a hybrid film (Fig. 1g). CNT film has good electrical conductivity, gas permeability, and conformal covering ability. As the top electrode of the device, it allows for the fast passage of water molecules, and is also conducive to the efficient collection and transmission of carriers. AAO membrane has controllable nanochannel structures (Fig. 1e, f and Fig. S1c, d). Due to the contribution of capillary pressure, the high density of these nanopores helps adsorbed water molecules to form condensed liquid in the pores. Compared with the messy porous structure, the oriented nanochannel structure increases the water slip length, thereby improving the ion transport efficiency.

According to the X-ray energy dispersive spectrum (EDS) element mapping as shown in Fig. S5 and S6, there is a large amount of oxygen distribution in AAO. Correspondingly, there should be a large number of hydroxyl groups on the surface of AAO. The high density of oxygen-containing functional groups makes it easy for moisture to penetrate into the nanochannels of AAO, and the high surface charge density increases ion selectivity. In addition, as the bottom electrode of the device, liquid metal has good conductivity and hydrophilicity. Such humidity-enabled electric generator (HEEG) can produce a continuous direct current (DC) output in a humid environment, as shown in Fig. 1h and 1i. Single unit with small size $\left(0.95 \mathrm{~cm}^{2}\right)$ can deliver a continuous $\mathrm{V}_{\mathrm{oc}}$ of $0.8-1.1 \mathrm{~V}$ and a gradually decayed $\mathrm{I}_{\mathrm{sc}}$ (the initial 
maximum value is of $1.5-2.5 \mu \mathrm{A}$ ) under an environment with temperature fluctuating around $25^{\circ} \mathrm{C}$ and relative humidity $(\mathrm{RH})$ fluctuating around $93 \%$. The gradually decayed current might be attributed to the inferior instability of oxygen functional groups of CNT under humid condition, as shown in Fig. S7b and Supplementary Table 2. The role of oxygen functional groups will be discussed in detail in the mechanism section. Both $\mathrm{V}_{\mathrm{OC}}$ and $\mathrm{I}_{\mathrm{SC}}$ last for at least one month (Fig. $1 \mathrm{~h}$ and $\mathrm{i}$ ), indicating that the power output is not a transient phenomenon, which is quite different from porous carbon films-based hydroelectric generator with pulsed electric output.

We argue that this power generation process is related to the rectification effect of nanopores(diode 2) and the built-in electric field induced ionization and charge transfer effect in the humidity-diode $1^{41}$, as shown in Fig. 1b. Generally, carriers in semiconductor and ions in solution are similar in many ways. In nanopores, the negatively charged surface of CNT has a high concentration of positive ions which can be regarded as carriers, and is similar to the P-type semiconductor. On the contrary, the positively charged AAO channel is similar to the $\mathrm{N}$-type semiconductor, and the presence of hydroxyl groups inducing the positive charged surface of AAO is verified by the Zeta potential and I-V sweeping measurement, which will be discussed later in Figure 3. Joining CNT and AAO results in a PN-like junction, and in the region close to the CNT/AAO interface, the ion transport phenomenon is mainly dominated by the ionic diodetype PN junction which is named humidity-diode $1^{41}$. In humidity-diode 1 , there is a strong built-in electric field in the depletion region of the PN junction ( $\vec{E}$ from AAO to CNT), and the adsorbed water molecules partially ionize hydrogen ions and hydroxide ions under the action of the built-in electric field. The hydrogen ions move in the direction of the built-in electric field and gather near the CNT, while the hydroxide ions shift in the opposite direction. On the other hand, since the thickness of AAO is hundreds of times that of CNT, in the AAO area far from the CNT/AAO interface, a reasonable assumption is that the interfacial molecular interactions between the surface of the AAO nanochannel and the ions flowing through them is dominated by the electrical double layer related Debye screening effect. Since the concentration of water molecules in the AAO channel is much higher near CNT than that near the In-Ga liquid metal electrode, this asymmetric concentration distribution can still effectively achieve nanopore rectification, which is equivalent to diode $2^{41}$. And the movable ions ionized in the built-in electric field of humidity-diode 1 are mainly concentrated on the CNT/AAO interface, which further strengthen the concentration asymmetry in diode 2 . Therefore, if the hybrid membrane is connected to the external circuit, as long as the moisture always exists, water molecules continuously adsorb, ionize, and selectively transport due to the synergy between humidity-diode 1 and diode 2 . For more detailed theoretical analysis, please see the supplementary material, and for more detailed experimental analysis, please refer to the mechanism section.

It is worth noting that only the CNT/liquid metal double-layer structure (without AAO) cannot produce any power output in a high-humidity environment. In order to further eliminate possible contribution through electrochemical reactions, removal of oxygen and nitrogen from the gas phase can still yield similar current and voltage generation (Fig. S8). Besides, the device was placed in a room temperature and high humid environment for cyclic voltammetry (CV) testing. The CV curve of the device is similar before and 
after three hours of operation, and there is no peak indicating the redox reaction of the interface active material as shown in Fig. S9. The above experimental results provide additional evidence that chemical corrosion in the AAO and redox reaction in electrode materials are not the source of energy of the observed electrical outputs.

\section{Electrical output performance of HEEG}

First, we study the influence of humidity on the HEEG performance, as shown in Fig. 2a and Fig. S10a. With the evolution of humidity ( $\mathrm{RH}$ of $11 \%-40 \%-70 \%-93 \%)$, more water molecules can be adsorbed, ionized, and selectively transported. Thus, $\mathrm{V}_{\mathrm{OC}}$ increases monotonically from $0.5 \mathrm{~V}$ to $1.05 \mathrm{~V}$, and $\mathrm{I}_{\mathrm{SC}}$ augments monotonically from around $28 \mathrm{nA}$ to $1.8 \mu \mathrm{A}\left(\mathrm{V}_{\mathrm{OC}}\right.$ and $\mathrm{I}_{\mathrm{SC}}$ are the average values calculated during the initial working period of $2 \mathrm{~h}$, the same as below). This output trend contrasts with that of protein nanowire-based $\mathrm{HEEG}^{33}$, which shows the highest $\mathrm{V}_{\mathrm{OC}}$ of $0.5 \mathrm{~V}$ at a $\mathrm{RH}$ of $40-50 \%$. The influence of the thickness of CNT films on device performance is displayed in Fig. 2b. When a CNT film is too thin, the decrease of initial concentrations of $\mathrm{COO}^{-}$in the CNT domain leads to a lower built-in potential $\mathrm{V}_{\mathrm{B}}$ in ionic diode-type AAO/CNT junction, and the electrode resistance of the thin CNT film becomes relatively large, which is not conducive to power generation. However, a CNT film that is too thick will prevent water molecules from entering the AAO nanochannel. Therefore, as the average thickness of a CNT film increases from $\sim 60 \mathrm{~nm}$ to $\sim 1400 \mathrm{~nm}$, both $\mathrm{V}_{\mathrm{OC}}$ and $\mathrm{I}_{\mathrm{SC}}$ measured in the experiments show a tendency to increase first and then become saturated (Fig. $2 \mathrm{~b}$ and Fig. S3). The hole size of the AAO nanochannel is another important factor, as shown in Fig. 2c. Since the Debye length is related to the ion concentration in the solution, it is usually $1-100 \mathrm{~nm}$. The exclusion-enrichment effect of ions requires that the smallest cross-sectional size in the nanopore channel be equal to or smaller than the Debye length ${ }^{42}$, so too large pore size is not conducive to power generation. On the other hand, the too small pore size causes the water molecules captured by the nanopores to be relatively limited. The optimal pore size turns out to be around $90 \mathrm{~nm}$.

Besides, the HEEG can be manufactured into devices with different working areas. Fig. $2 \mathrm{~d}$ and Fig. S10b show that $\mathrm{V}_{\mathrm{OC}}$ varies between 0.8-1.1V for different areas and $\mathrm{I}_{\mathrm{SC}}$ increases as the area of the device augments. When the area of a single device reaches $314 \mathrm{~mm}^{2}$, short-circuit current $\mathrm{I}_{\mathrm{SC}}$ is $\sim 7.7 \mu \mathrm{A}$. It is worth noting that the $I_{S C}$ density increases monotonically as the device area decreases, as shown in Fig. S10c. When the device area is as small as $1 \mathrm{~mm}^{2}$, the maximum short-circuit current density is $11.3 \mu \mathrm{Acm}^{-2}$. One possible explanation is that a smaller device area is accompanied by fewer defects and a higher carrier collection efficiency of the electrode. Fig. $2 e$ and $2 f$ display the power output performance under resistive load conditions for a device with $1 \mathrm{~mm}^{2}$ working area. The best resistive load is $10 \mathrm{M} \Omega$, and the power density is as high as $1.3 \mu \mathrm{Wcm}^{-2}$ and $277 \mu \mathrm{Wcm}^{-3}$. In addition, the output $\mathrm{V}_{\mathrm{OC}}$ of the hybrid membrane has little decay after being placed in $93 \% \mathrm{RH}$ environment for 30 days and ambient environment for another 60 days. (Fig. S11a). Thanks to the strong hydrogen bonds and van der Waals forces at the interface between CNT films and AAO membranes, robust mechanical frameworks are provided to handle the deformation of water-related capillary and external distortion, as shown in Fig. 
S11b. The device has high output power density and long-term stability at the same time, and its performance compared with devices of the same type reported in the literature is shown in Supplementary Table 3.

\section{Analysis of mechanism and influence factors}

The asymmetric hybrid membrane gives rise to a nonlinear (ionic diode-type) current in a humid environment as shown in Fig. 3a and 3b, which means the ion conduction ability is different under positive and negative voltage. Under the forward voltage, the applied bias voltage is opposite to the direction of the built-in electric field formed at the AAO/CNT interface, which weakens the built-in potential in humidity-diode 1 and reduces the production rate of aquatic charged ions. And in diode 2 , AAO has a narrow pore size distribution and rich positive surface charges, the Debye screening effect prevents $\mathrm{H}^{+}$ions from entering the nanopores, and the $\mathrm{OH}^{-}$existing in the pores are squeezed out against the direction of the applied bias. The final result is that the ions in the nanopores are evacuated, which greatly increases the impedance of the nanopores. Under reverse voltage, the applied bias voltage is in the same direction as the built-in electric field formed at the AAO/CNT interface, which enhances the builtin potential in humidity-diode 1 and improves the generation rate of aquatic charged ions. And the Debye screening effect will promote the $\mathrm{OH}^{-}$ions to enter the pores, so that the impedance of the nanopores is reduced. Therefore, we attribute this unique ionic current rectification in a humid environment to the synergistic effect of diode 1 and diode 2. Moreover, this rectification effect is observed in both high humidity $(93 \% \mathrm{RH})$ and low humidity $(11 \% \mathrm{RH})$ test environments, as shown in Fig. 3a and $3 \mathrm{~b}$.

Ion rectification effect improves energy conversion efficiency due to steady-state one-way ion transport, and is highly dependent on surface charge density. We use oxygen plasma treatment to study the effect of surface charge density of AAO and CNT on device performance. As shown in Fig. $3 \mathrm{c}$ and $3 \mathrm{~d}$, as the $\mathrm{pH}$ increases from 4.00 to 10.00 , CNT's zeta potential is always negative and its absolute value gradually increases, indicating that CNT is negatively charged and plasma treatment augments the surface charge density of CNT. The negative charge should be ascribed to the carboxyl groups of CNT. AAO nanochannels contain a large number of amphoteric -OH groups, and their surface polarity is $\mathrm{pH}$ dependent. Indeed, CNT release free protons under moisture, leading to initial acidic environment. Under suitable acidic conditions, the surface polarity of AAO channel is positively charged, and plasma treatment is conducive to the establishment of positive surface polarity. Let us compare four different functional materials plasma treating methods: (i) neither CNT nor AAO with plasma treating; (ii) just AAO with air plasma treating; (iii) both with air plasma treating; (iv) both with oxygen plasma treating. Among these processing methods, both CNT and AAO with oxygen plasma treating own the best performance, and the $\mathrm{V}_{\mathrm{OC}}$ and $\mathrm{I}_{\mathrm{SC}}$ elevate $30 \%$ and $700 \%$ respectively compared to that of neither with plasma treating, as shown in Fig. 3e. The effect of plasma treating time has also been demonstrated as depicted in Fig. S12. Above experimental results indicate the key role of surface charge density on the device efficiency. In addition, the use of vacuum dehumidification to prevent the dynamic adsorption of water molecules at the AAO/CNT interface depletes the electrical output, while the removal of dehumidification restores the continuous output, as shown in Fig. 4a. By increasing the relative humidity, the environment provides 
more water molecules, and the electrical output of the device is correspondingly increased, as shown in Fig. 4b, indicating that the environmental humidity is the energy input source of the power generation device.

According to the aforementioned results, a feasible mechanism of electricity generation is illustrated in Fig. $3 \mathrm{f}$ and $3 \mathrm{~g}$. Since the top electrode area is exposed to moisture air and the bottom electrode area is sealed, moisture is first adsorbed on the CNT/AAO interface and become ionized. Due to the contribution of capillary pressure, the adsorbed water molecules form a condensed liquid in the nanopores to facilitate ion transport. Under the action of an electric field (directed from AAO points to CNT) built in the depletion region of the PN junction, water molecules accelerate ionization into movable hydrogen ions and hydroxide ions. Among them, the hydrogen ions move in the direction of the built-in electric field, and the hydroxide ions move in the opposite direction. Due to the high selectivity of the AAO positively charged nanochannel under the Debye screening effect, the hydroxide ions accelerated by the built-in electric field continue to move along the direction of the nanochannel to the bottom electrode. Ambient environment continuously supplies water molecules, and the successive water molecule adsorption, ionization and ion selective directional transportation generate a sustained electric output. The device seems to display a particularly efficient charge transfer for continuous electric output, probably because of a synergy between the ion rectification of nanofluidic diode and the built-in electric field induced ionization and charge transfer. However, it is still not clear how the ion current inside the device and the electronic current in the load resistance can be converted to each other. The traditional streaming potential usually relies on the oxidation-reduction reaction of the electrode to convert the ionic current into a continuous electronic current $^{39,40,43}$. However, as mentioned before and shown in Fig. S8 and S9, the interference of redox is excluded. One possible conversion mechanism is based on the ionovoltaic phenomenon ${ }^{44}$ which still requires further investigation. It is worth noting that when the bottom electrode In-Ga liquid metal is replaced by an inert Au electrode, the device $\mathrm{V}_{\mathrm{OC}}$ drops from $1.1 \mathrm{~V}$ to $0.26 \mathrm{~V}$ in Fig. 4c. One possible explanation is that asymmetric CNT ( -4.7eV), AAO (Al: $-4.28 \mathrm{eV}$, aluminum oxide: $-0.9 \sim-2.9 \mathrm{eV}$ ) and In-Ga (In: $-4.12 \mathrm{eV}, \mathrm{Ga}:-4.2 \mathrm{eV}$ ) with suitable work function leads to effective charge collection of holes and electrons. The work function of $\mathrm{Au}$ is $-5.1 \mathrm{eV}$, which may not match with the movement of the carriers, decreasing the final voltage output ${ }^{29}$. We applied airflow disturbances of different magnitudes to the device in a high-humidity environment, and observed the changes of HEEG electrical signals. The device voltage and current performance show no obvious response to varied airflow flow speeds as shown in Fig. 4d and Fig. S13. This phenomenon indicates that our power generation mechanism is quite different from those devices which are dominated by water evaporation process. It also proves that the HEEG device has reliable working stability and excellent environmental adaptability.

\section{Application of HEEG}

Connecting multiple units in series and parallel to supply power to external circuit loads is of great importance to practical applications. Here we use eighteen devices, under the ambient condition $\left(\sim 65 \% \mathrm{RH}, 25^{\circ} \mathrm{C}\right)$, without external energy storage device and current rectification apparatus. The seriesparallel combination directly lights up ten LED lights at the same time, and can also powers the electronic 
clock, as shown in Fig. 5a top and middle, respectively. The circuit diagram is shown in Fig. 5a bottom. This power generation device can also be used in self-powered breathing monitoring scenarios, as shown in Figure $5 \mathrm{~b}$, the inset is a partial enlarged view of the generated current signal. Regular breathing of about $0.5 \mathrm{~Hz}$ is reliably monitored. In addition, eight power generating units connected in series charge a $47 \mu \mathrm{F}$ capacitor to $2.7 \mathrm{~V}$ within 30 minutes in Fig. $5 \mathrm{c}$. Different voltage values achieved by connecting different numbers of device units in series exhibit a good linear relationship between the voltage and the number of series units from the fitted curve of Fig. $5 \mathrm{~d}$. Twenty power generating units connected in series generate $\mathrm{a} \mathrm{V}_{\mathrm{OC}}$ of $\sim 18 \mathrm{~V}$.

\section{Conclusion}

In this work, we have integrated the ionic diode-type hybrid membrane design into the nanofluid energy conversion system, and demonstrated a hydroelectric power generation device that continuously collects energy from environmental moisture. Through material selection and device structure design, our devices simultaneously meet the requirements of rapid capture of water molecules, high-efficiency ionization of water molecules, high ion conductance, selective directional transport of ions, highly effective conversion between ion current and electron current. The devices elaborated in the present work provide a DC voltage and current output with maximum $\mathrm{V}_{\mathrm{OC}}$ and $\mathrm{I}_{\mathrm{SC}}$ of up to $1.1 \mathrm{~V}$ and $7.7 \mu \mathrm{A}$ for a single unit, and the maximum short circuit current density and power output density reach $11.3 \mu \mathrm{Acm}^{-2}$ and $1.3 \mu \mathrm{Wcm}^{-2} / 277 \mu \mathrm{Wcm}^{-3}$. Most importantly, compared with most previously reported nanostructured devices whose power generation is drastically degraded after working for a few hours or days, our hydropower devices have shown continuous electrical output for at least one month. Through experimental design and theoretical analysis, the power output of our devices has a strong correlation with the ion rectification phenomenon of nanofluidic diode and with the built-in electric field induced ionization and charge transfer. In addition, the problem of air flow interference in practical applications has also been considered. Commercial LED displays can be easily driven by connecting multihydroelectric devices in series and parallel. In addition, self-powered breathing monitoring application has been explored. Our work paves a new route to collecting energy from environmental moisture, promotes a better understanding of ion transport in confined nanospace and provides a universal effective way for the power supply of low-power loT devices.

\section{Declarations}

\section{Acknowledgements}

We acknowledge the financial support from the National Natural Science Foundation of China (Grant Nos. 51802293, 11890673) and Fundamental Research Funds for the Central Universities of China (Grant Nos. SWJTU 2682021CX120). This work was completed at the Research Center for Ultra-precision Surface Manufacturing, Southwest Jiaotong University. We sincerely thank Linmao Qian and Bingjun Yu 
for providing scientifically standardized experimental sites and experimental environment, we also thank Zhongbao Jiang, Changliu He, Pengfei Zhu, Lingxu Jia for their daily discussion.

\section{Author contributions}

Y. Z., T. Y. designed the research and wrote the paper. T.Y. and Q. H. direct this research. Y. Z. conducted the majority of experimental work. T. Y. proposed mechanisms. K. S. analyzed data and discussed results. F. G., Y. S. and S.C. prepared CNT films. L. C. tested the thickness of CNT film. X. L., assisted in setting up test equipment and gave suggestions on applications. J. Z. and C. F., completed the electron micrograph shooting.

\section{Competing interests}

The authors declare no competing interests.

\section{References}

1. Xu, W. et al. A droplet-based electricity generator with high instantaneous power density. Nature $\mathbf{5 7 8}$, 392-396, (2020).

2. Nie, J. et al. Power generation from the interaction of a liquid droplet and a liquid membrane. Nat. Commun. 10, 2264, (2019).

3. Qin, Y. et al. Constant Electricity Generation in Nanostructured Silicon by Evaporation-Driven Water Flow. Angew. Chem. Int. Ed. 59, 10619-10625, (2020).

4. Ding, T. et al. All-Printed Porous Carbon Film for Electricity Generation from Evaporation-Driven Water Flow. Adv. Funct. Mater. 27, 1700551 (2017).

5. Yang, P. et al. Solar-driven simultaneous steam production and electricity generation from salinity. Energy Environ. Sci. 10, 1923-1927, (2017).

6. Zhou, X. et al. Harvesting Electricity from Water Evaporation through Microchannels of Natural Wood. ACS Appl. Mater. Interfaces 12, 11232-11239, (2020).

7. Ma, Q. et al. Rational Design of MOF-Based Hybrid Nanomaterials for Directly Harvesting Electric Energy from Water Evaporation. Adv. Mater. 32, 2003720, (2020).

8. Xue, G. et al. Water-evaporation-induced electricity with nanostructured carbon materials. Nat. Nanotechnol. 12, 317-321, (2017).

9. Li, M. et al. Biological Nanofibrous Generator for Electricity Harvest from Moist Air Flow. Adv. Funct. Mater. 29, 1901798, (2019).

10. Li, L. et al. Sustainable and flexible hydrovoltaic power generator for wearable sensing electronics. Nano Energy 72, 104663, (2020).

11. Das, S. S., Pedireddi, V. M., Bandopadhyay, A., Saha, P. \& Chakraborty. S. Electrical power generation from wet textile mediated by spontaneous nanoscale evaporation. Nano Lett. 19, 7191-7200 (2019). 
12. Yun, T. G., Bae, J., Rothschild, A. \& Kim, I.-D. Transpiration driven electrokinetic power generator. ACS Nano 13, 12703-12709 (2019).

13. Zhang, R. et al. A streaming potential/current-based microfluidic direct current generator for selfpowered nanosystems. Adv. Mater. 27, 6482-6487 (2015).

14. Yin, J. et al. Generating electricity by moving a droplet of ionic liquid along graphene. Nat. Nanotechnol. 9, 378-383, (2014).

15. Kral, P. \& Shapiro, M. Nanotube Electron Drag in Flowing Liquids. Phys. Rev. Lett. 86, 131-134, (2001).

16. Yang, S. et al. Mechanism of Electric Power Generation from Ionic Droplet Motion on Polymer Supported Graphene. J. Am. Chem. Soc. 140, 13746-13752, (2018).

17. Yin, J. et al. Waving potential in graphene. Nat. Commun. 5, 3582, (2014).

18. Fei, W. et al. Waving potential at volt level by a pair of graphene sheets. Nano Energy $60,656-660$ (2019).

19. Zhu, G. et al. Harvesting water wave energy by asymmetric screening of electrostatic charges on a nanostructured hydrophobic thin-film surface. ACS Nano 8, 6031-6037 (2014).

20. Zhao, X. J., Kuang, S. Y., Wang, Z. L. \& Zhu, G. J. A. n. Highly adaptive solid-liquid interfacing triboelectric nanogenerator for harvesting diverse water wave energy. ACS Nano 12, 4280-4285 (2018).

21. Kwak, S. S. et al. Triboelectrification-Induced Large Electric Power Generation from a Single Moving Droplet on Graphene/Polytetrafluoroethylene. ACS Nano 10, 7297-7302, (2016).

22. $\mathrm{Xu}, \mathrm{W}$. et al. SLIPS-TENG: robust triboelectric nanogenerator with optical and charge transparency using a slippery interface. National Science Review 6, 540-550, (2019).

23. Shen, D. et al. Self-Powered Wearable Electronics Based on Moisture Enabled Electricity Generation. Adv. Mater. 30, 1705925, (2018).

24. He, D. et al. Electricity generation from phase-engineered flexible MoS2 nanosheets under moisture. Nano Energy 81, 105630, (2021).

25. Li, L. et al. Moisture-Driven Power Generation for Multifunctional Flexible Sensing Systems. Nano Lett. 19, 5544-5552, (2019).

26. Long, Y. et al. Moisture-induced autonomous surface potential oscillations for energy harvesting. Nat. Commun. 12, 5287, (2021).

27. Zhao, F. et al. Highly efficient moisture-enabled electricity generation from graphene oxide frameworks. Energy Environ. Sci. 9, 912-916 (2016).

28. Cheng, H. et al. Spontaneous power source in ambient air of a well-directionally reduced graphene oxide bulk. Energy Environ. Sci. 11, 2839-2845, (2018).

29. Huang, Y. et al. Interface-mediated hygroelectric generator with an output voltage approaching 1.5 volts. Nat. Commun. 9, 4166, (2018). 
30. Radha, B. et al. Molecular transport through capillaries made with atomic-scale precision. Nature 538, 222-225 (2016).

31. Gopinadhan, K. et al. Complete steric exclusion of ions and proton transport through confined monolayer water. Science 363, 145-148 (2019).

32. Mouterde, T. et al. Molecular streaming and its voltage control in ångström-scale channels. Nature 567, 87-90 (2019).

33. Liu, X. et al. Power generation from ambient humidity using protein nanowires. Nature $578,550-554$, (2020).

34. Bae, J., Yun, T. G., Suh, B. L., Kim, J. \& Kim, I.-D. Self-operating transpiration-driven electrokinetic power generator with an artificial hydrological cycle. Energy Environ. Sci. 13, 527-534, (2020).

35. Xu, T. et al. An efficient polymer moist-electric generator. Energy Environ. Sci. 12, 972-978, (2019).

36. Wang, H. et al. Bilayer of polyelectrolyte films for spontaneous power generation in air up to an integrated 1,000 V output. Nat. Nanotechnol. 16, 811-819, (2021).

37. Kim, Y. D., Choi, S., Kim, A. \& Lee, W. Ionic Current Rectification of Porous Anodic Aluminum Oxide (AAO) with a Barrier Oxide Layer. ACS Nano 14, 13727-13738, (2020).

38. Zhu, X. et al. Unique ion rectification in hypersaline environment: A high-performance and sustainable power generator system. Sci. Adv. 4, eaau1665 (2018).

39. Kim, S. et al. Neuromorphic van der Waals crystals for substantial energy generation. Nat. Commun. $12,47,(2021)$.

40. Xin, W. et al. High-performance silk-based hybrid membranes employed for osmotic energy conversion. Nat. Commun. 10, 3876, (2019).

41. Cheng, L. J., Guo, L. J. Nanofluidic diodes. Chem. Soc. Rev. 39, 923-938 (2010).

42. Schoch, R. B., Han, J. \& Renaud, P. Transport phenomena in nanofluidics. Rev. Mod. Phys. 80, 839883, (2008).

43. Li, L. et al. A novel, flexible dual-mode power generator adapted for wide dynamic range of the aqueous salinity. Nano Energy 85, 105970 (2021).

44. Yoon, S. G. et al. Natural evaporation-driven ionovoltaic electricity generation. ACS Appl. Electron. Mater. 1, 1746-1751 (2019).

\section{Figures}



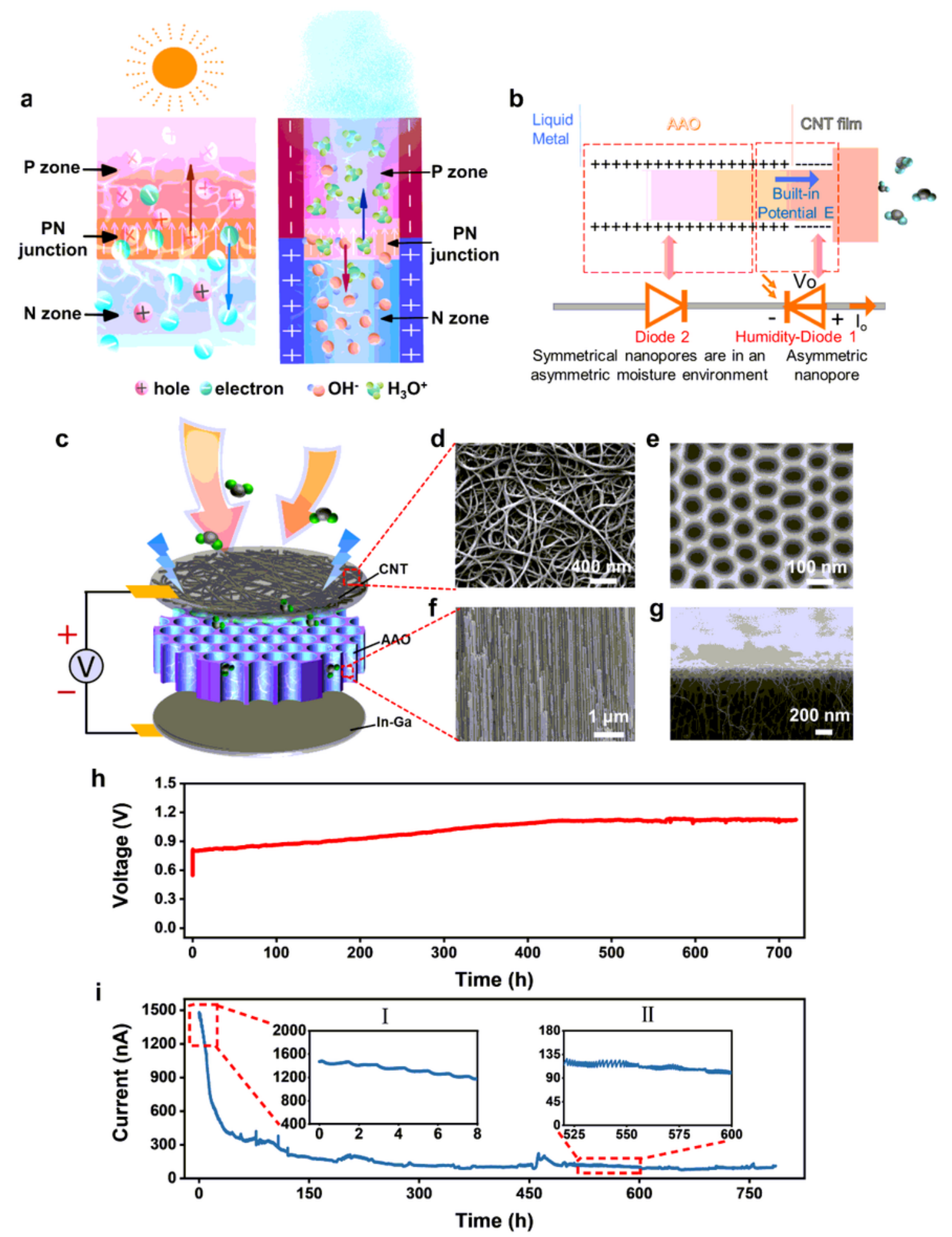

\section{Figure 1}

Ionic diode-type hybrid membrane device and the electric performance. a) Schematic diagram of solar photovoltaic power generation with PN junction, illustration of hydrovoltaic power generation principle inspired by photovoltaic effect. b) Schematic diagram of the working principle for HEEG, the darker the color, the greater the humidity, the circuit symbol of Humidity-Diode1 refers to the representation method of the Photo-Diode. c) Explosion view of the HEEG. The SEM images of CNT top electrode d), AAO from 
the top view e) and AAO from the lateral view f). g) The SEM image of the contact interface between CNT and AAO. h), i) The HEEG continuously work over 700 hours for voltage and current respectively in $93 \% \mathrm{RH}, 25^{\circ} \mathrm{C}$ environment, indicating the robust and stable continuous output performance.

a

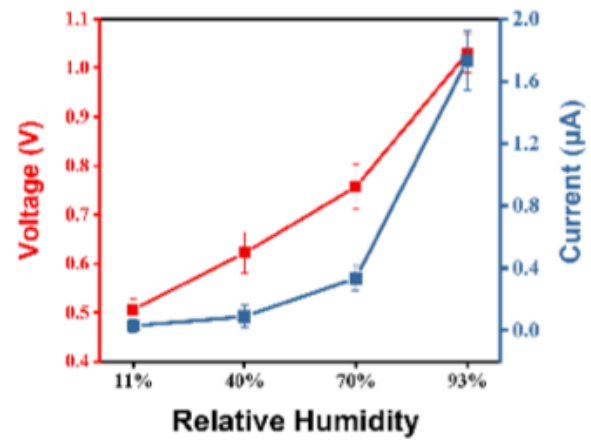

d

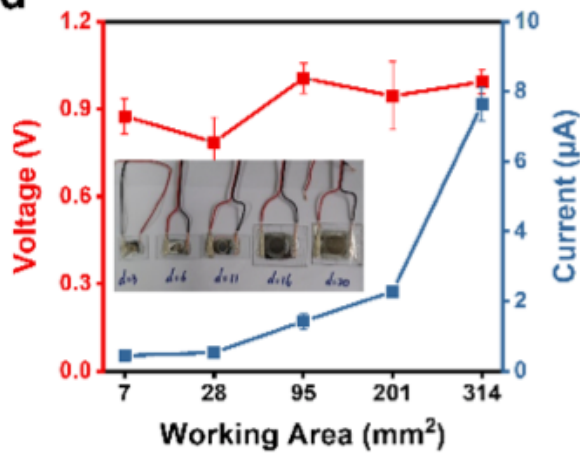

b

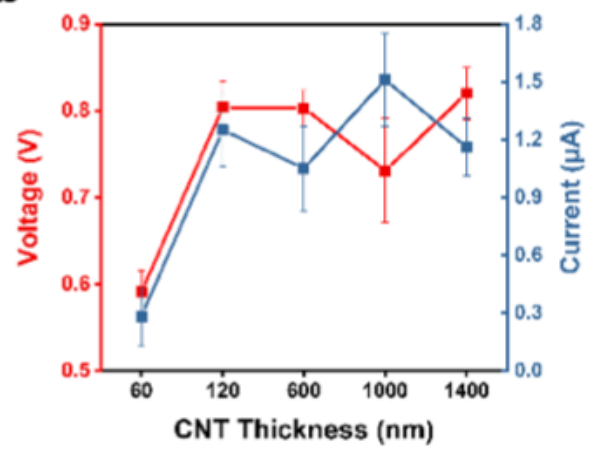

e

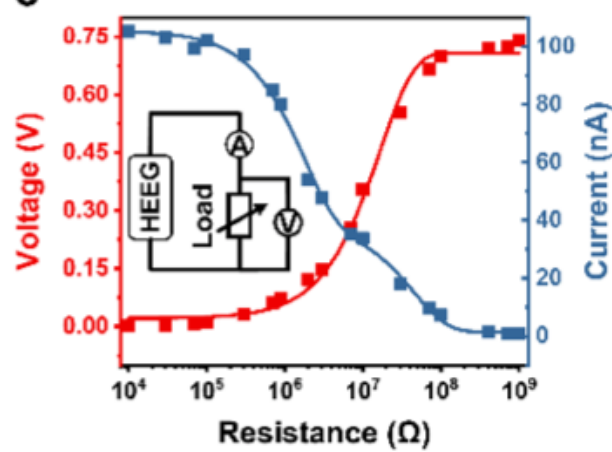

C

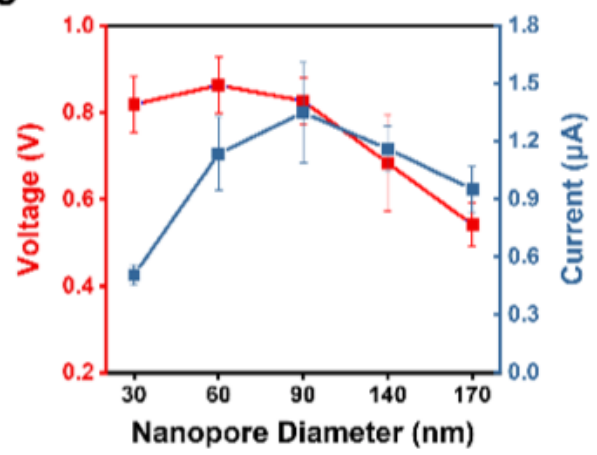

f

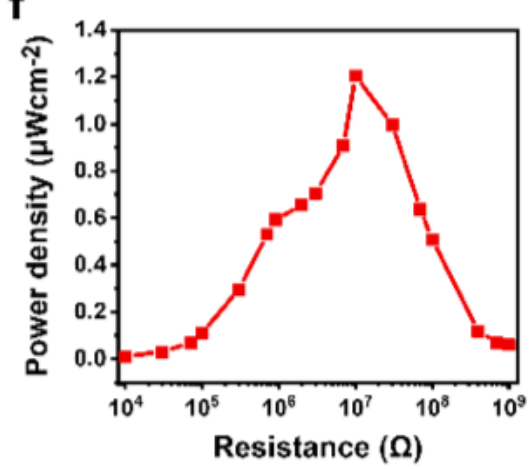

Figure 2

Electric output performance of HEEG and the corresponding influence factors. Influence of relative humidity a), CNT thickness b), nanopore size c) and device working area d) to VOC, ISC, and the inset image in d) is the device optical photos for different working areas. e) Current and voltage change with different load resistance for the device which has a working area of $1 \mathrm{~mm} 2$. f) Corresponding output power density changes with different load resistance, and when external resistance is $10 \mathrm{M} \Omega$, the device has the best output performance. 
a
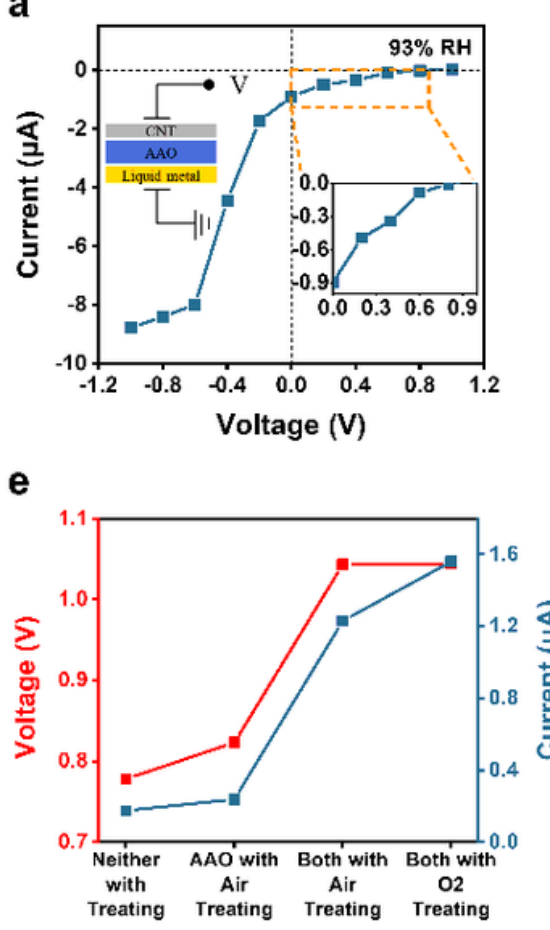

Plasma Treating Methods
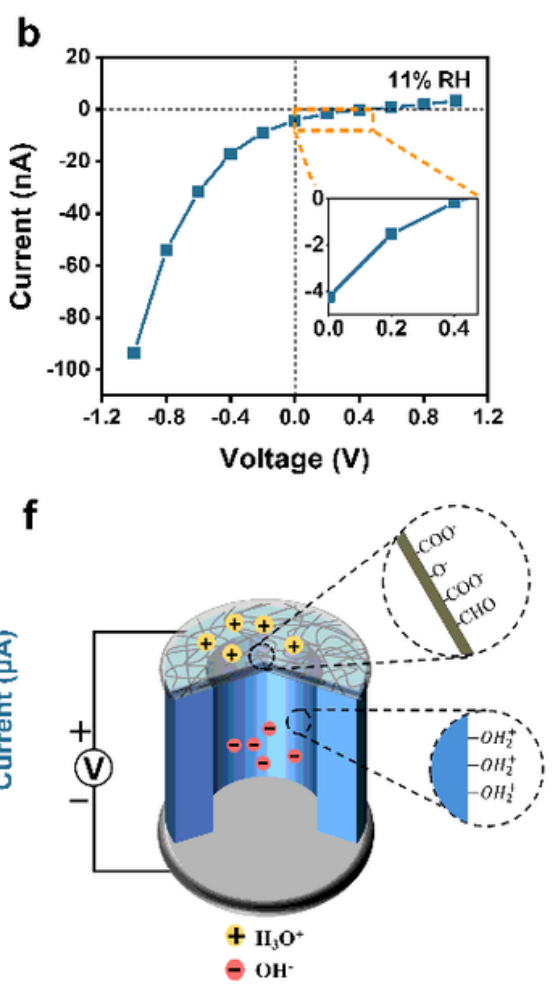

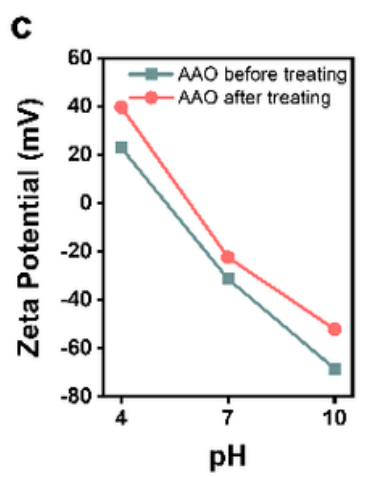

d

g

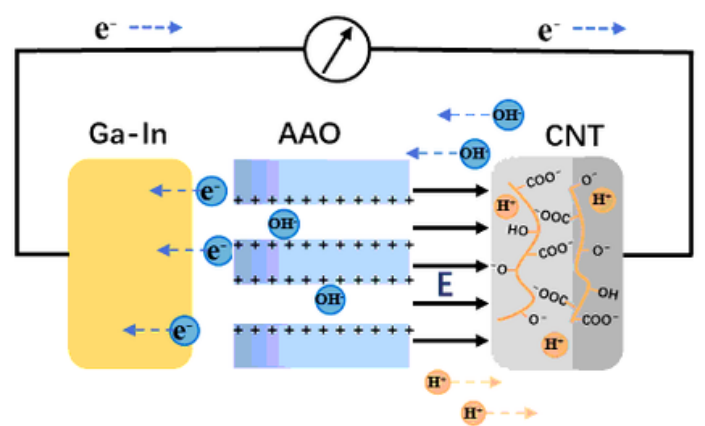

\section{Figure 3}

Mechanism analysis of the HEEG. a) b) I-V sweeping curves for device in high and low RH condition, which effectively demonstrate the ion-diode characteristics for unidirectional electrical conduction. c) d) Surface Zeta potential of CNT film and AAO membrane in different $\mathrm{pH}$ condition before and after $\mathrm{O}$ plasma treating, respectively, verifying effective surface modification. e) Result of device's VOC, ISC for four different plasma treating methods, and the HEEG shows the best performance after both membrane material being treated with oxygen plasma. f) Cartoon shows the functional groups existing on the CNT and AAO surface. g) Schematic depiction of the harvesting moisture energy process. 
$\mathbf{a}$

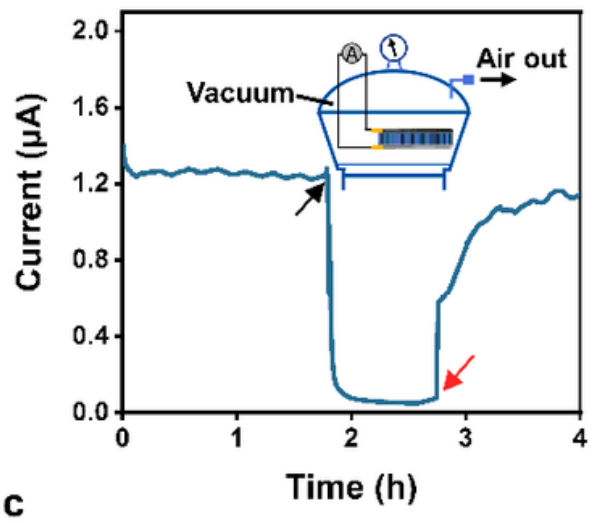

c

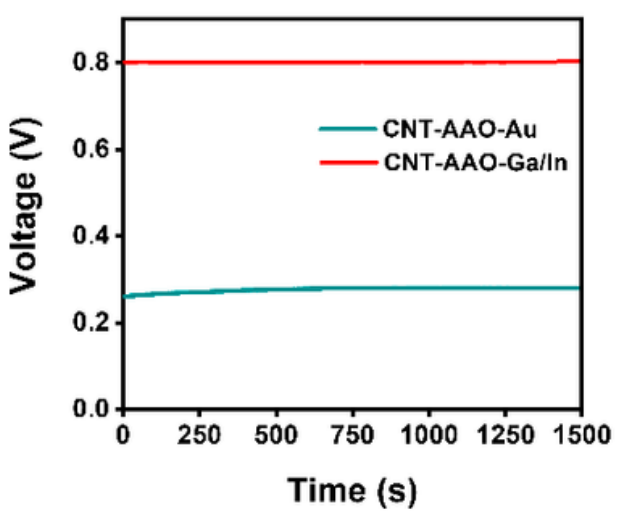

b
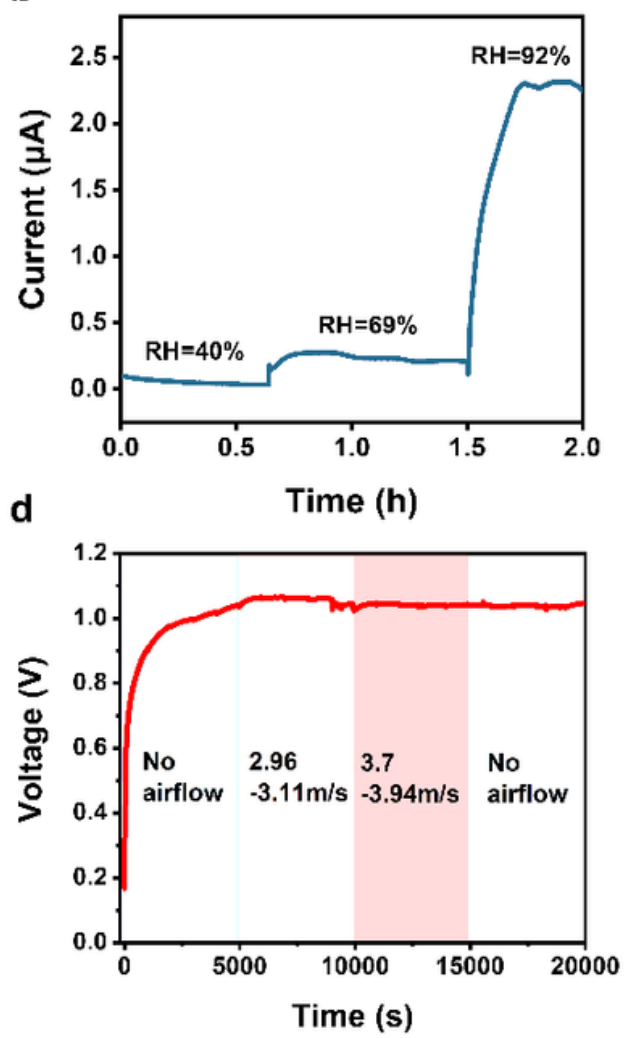

Figure 4

Electrical output of HEEG under different conditions. a) When the moisture (test condition $25^{\circ} \mathrm{C}, 93 \% \mathrm{RH}$ ) in the container is pumped away (inset), the continuous current output of HEEG from the surrounding environment is destroyed (black arrow). Until the moisture is refilled, the current begins to return to its original value (red arrow). b) The current changes continuously with the relative humidity. c) Voltage comparison between CNT-AAO-In/Ga and CNT-AAO-Au. When liquid metal is replaced by gold, power output can still be generated. d) When HEEG is exposed to the airflow environment of different wind speeds, its voltage performance has no obvious change, indicating that the airflow has little effect on the HEEG voltage signal. 
a

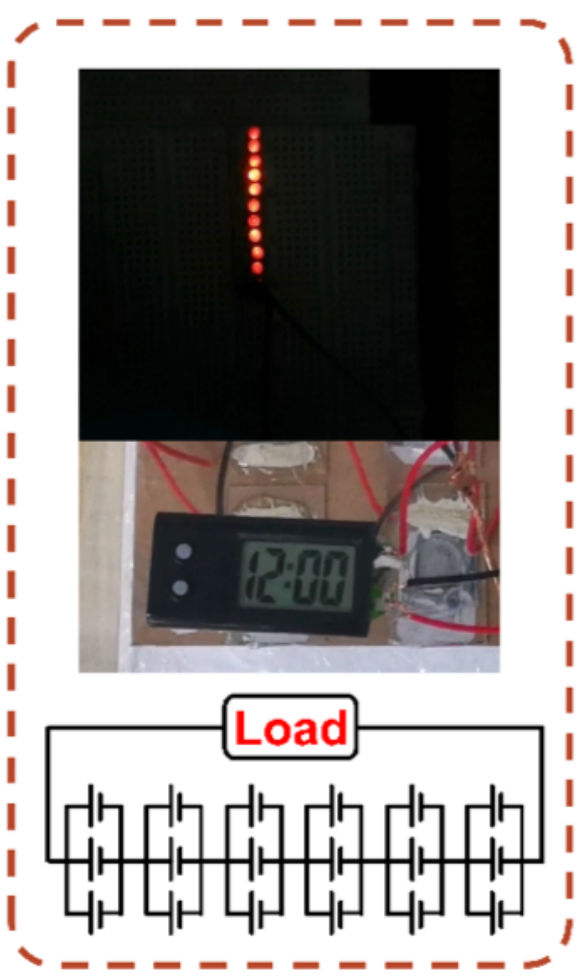

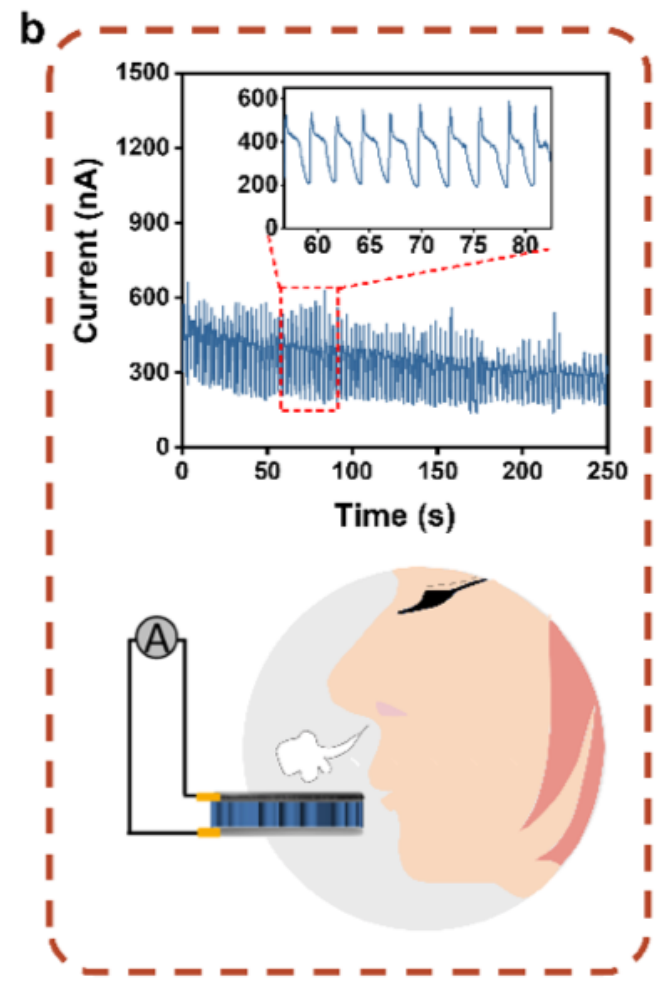
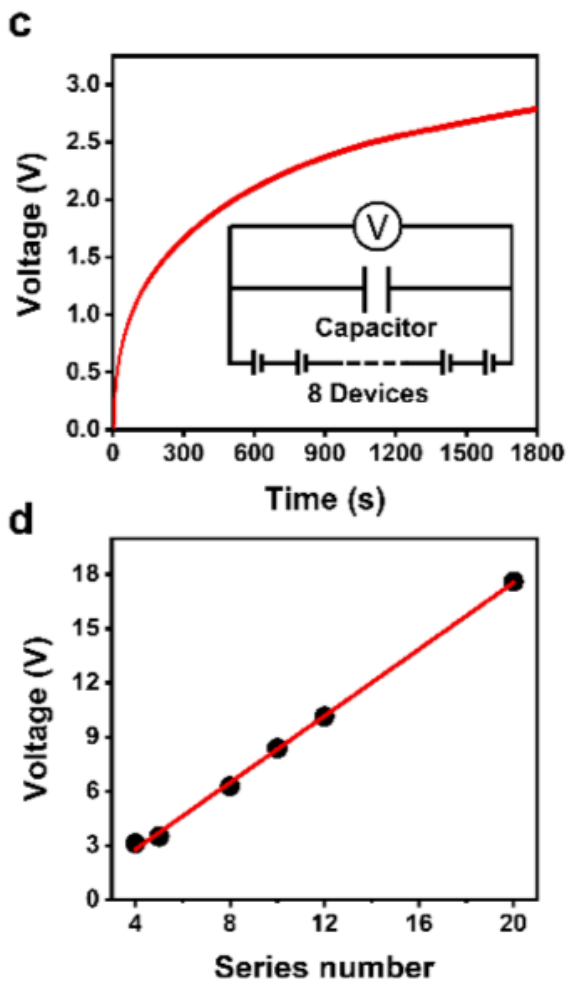

\section{Figure 5}

Application of HEEG devices. a) Combination of 18 devices in series and parallel connection can light up ten LEDs and power up a digital electronic watch directly under laboratory environment $\sim 65 \% \mathrm{RH}, 25^{\circ} \mathrm{C}$.

b) Self-powered breathing monitoring application and its signal display, the bottom is the test schematic diagram. c) 8 units in series charge a $47 \mu \mathrm{F}$ capacitor to $\sim 2.8 \mathrm{~V}$ in $30 \mathrm{~min}$. d) The open-circuit voltage of different number of devices that are connected in series.

\section{Supplementary Files}

This is a list of supplementary files associated with this preprint. Click to download.

- SupportInformation20211116.docx 